\title{
Managing retinal vein occlusion
}

In boxes 1-3 of this Clinical Review by Christine A Kiire and N Victor Chong (BMJ 2012;344:e499, doi:10.1136/bmj.e499) reference 1 , not reference 2 , should have been cited after the box titles. In addition, the word "lower" should have been removed from the last sentence of the main text so that the sentence reads: "A small prospective study of 18 eyes with macular oedema as a result of branch retinal vein occlusion, in which eyes were randomised to receive treatment with either intravitreal bevacizumab injections alone or in combination with laser treatment, found that combined treatment reduced the number of reinjections."

Cite this as: $B M J$ 2012;344:e2110

๑ BMJ Publishing Group Ltd 2012 